\title{
Descent on toric fibrations
}

\author{
Alexei N. Skorobogatov
}

\begin{abstract}
We describe descent on families of torsors of a constant torus. A recent result of Browning and Matthiesen then implies that the Brauer-Manin obstruction controls the Hasse principle and weak approximation when the ground field is $\mathbb{Q}$ and the singular fibres are all defined over $\mathbb{Q}$.
\end{abstract}

\section{Introduction}

Let $T$ be a torus over a number field $k$. Let $X$ be a smooth, proper, geometrically integral variety with a surjective morphism $f: X \rightarrow \mathbb{P}_{k}^{1}$ whose generic fibre $X_{k(t)}$ is geometrically integral and is birationally equivalent to a $k(t)$-torsor of $T$. The main result of this note says that the set $X(k)$ is dense in $X\left(\mathrm{~A}_{k}\right)^{\mathrm{Br}}$ if certain auxiliary varieties satisfy the Hasse principle and weak approximation.

These varieties are given by explicit equations. Choose $\mathbb{A}_{k}^{1} \subset \mathbb{P}_{k}^{1}$ so that the fibre of $f$ at $\infty=\mathbb{P}_{k}^{1} \backslash \mathbb{A}_{k}^{1}$ is smooth. Let $P_{1}, \ldots, P_{r}$ be closed points of $\mathbb{P}_{k}^{1}$ such that each fibre of the restriction of $f$ to $\mathbb{P}_{k}^{1} \backslash\left(P_{1} \cup \ldots \cup P_{r}\right)$ is split, i.e. contains an irreducible component of multiplicity 1 that is geometrically irreducible. By a well known result (Lemma 3.1) the fibre $X_{P_{i}}$, for $i=1, \ldots, r$, has an irreducible component of multiplicity 1 . We fix such a component in each $X_{P_{i}}$ and define $k_{i}$ as the algebraic closure of the residue field $k\left(P_{i}\right)$ in the function field of this component. For $i=1, \ldots, r$ let $p_{i}(t) \in k[t]$ be the monic irreducible polynomial such that $P_{i}$ is the zero set of $p_{i}(t)$ in $\mathbb{A}_{k}^{1}$, and let $a_{i}$ be the image of $t$ in $k\left(P_{i}\right)=k[t] /\left(p_{i}(t)\right)$. Let $u, v$ be independent variables, and let $z_{i}$ be a $k_{i}$-variable, for $i=1, \ldots, r$. For $\alpha=\left\{\alpha_{i}\right\}$, where $\alpha_{i} \in k\left(P_{i}\right)^{*}$, we define the quasi-affine variety $W_{\alpha} \subset \mathbb{A}_{k}^{2} \times \prod_{i=1}^{r} R_{k_{i} / k}\left(\mathbb{A}_{k_{i}}^{1}\right)$ by

$$
\alpha_{i}\left(u-a_{i} v\right)=N_{k_{i} / k\left(P_{i}\right)}\left(z_{i}\right), \quad(u, v) \neq(0,0), \quad i=1, \ldots, r
$$

The varieties $W_{\alpha}$ are smooth and geometrically irreducible. Over an algebraic closure of $k$ such a variety is given by $\sum_{i=1}^{r}\left[k\left(P_{i}\right): k\right]$ equations in $2+\sum_{i=1}^{r}\left[k_{i}: k\right]$ variables. We can now state our main result.

Theorem 1.1 Suppose that for each $\alpha \in \prod_{i=1}^{r} k\left(P_{i}\right)^{*}$ the variety $W_{\alpha}$ satisfies the Hasse principle and weak approximation. Then $X(k)$ is dense in $X\left(\mathrm{~A}_{k}\right)^{\mathrm{Br}}$. 
The results of this kind are obtained by the descent method of Colliot-Thélène and Sansuc, and have a long history. When the relative dimension and the number of singular geometric fibres of $f$ are small, geometric proofs of the Hasse principle and weak approximation for $W_{\alpha}$ were obtained by Colliot-Thélène, Sansuc, SwinnertonDyer and others, see [7, 4, 19, 24, 9] and [21, Ch. 7]. The analytic tool in these proofs is Dirichet's theorem on primes in an arithmetic progression. Over $k=\mathbb{Q}$ one can do more if one uses analytic methods: the circle method [16, 8], sieve methods [1, 10] and recent powerful results from additive combinatorics [11, 12, 13, 3, 15, 22, Note that the circle method can sometimes be applied over arbitrary number fields, see [23, 18].

When $k=k\left(P_{1}\right)=\ldots=k\left(P_{r}\right)=\mathbb{Q}$, a recent result of Browning and Matthiesen obtained by methods of additive combinatorics [2, Thm 1.3] establishes the Hasse principle and weak approximation for $W_{\alpha}$. Hence we deduce the following corollary of Theorem 1.1.

Corollary 1.2 Let $X$ be a smooth, proper, geometrically integral variety over $\mathbb{Q}$, and let $f: X \rightarrow \mathbb{P}_{\mathbb{Q}}^{1}$ be a surjective morphism satisfying the following properties.

(a) There is a torus $T$ over $\mathbb{Q}$ such that the generic fibre $X_{\mathbb{Q}(t)}$ of $f$ is birationally equivalent to a $\mathbb{Q}(t)$-torsor of $T \times \mathbb{Q} \mathbb{Q}(t)$.

(b) There exists a finite subset $E \subset \mathbb{P}_{\mathbb{Q}}^{1}(\mathbb{Q})$ such that $X \backslash f^{-1}(E) \rightarrow \mathbb{P}_{\mathbb{Q}}^{1} \backslash E$ has split fibres.

Then $X(\mathbb{Q})$ is dense in $X\left(\mathrm{~A}_{\mathbb{Q}}\right)^{\mathrm{Br}}$.

This generalises a recent result due to A. Smeets, namely the unconditional counterpart of [22, Thm. 1.1, Rem. 1.3]. For a higher-dimensional version of this statement see Proposition 3.4.

For a number field $k$ of degree $n=[k: \mathbb{Q}]$ we write $\mathbf{x}=\left(x_{1}, \ldots, x_{n}\right)$ and denote by $N_{k / \mathbb{Q}}(\mathbf{x})$ the norm form $\operatorname{Norm}_{k / \mathbb{Q}}\left(x_{1} \omega_{1}+\ldots+x_{n} \omega_{n}\right)$, where $\omega_{1}, \ldots, \omega_{n}$ is a basis of $k$ as a vector space over $\mathbb{Q}$. The following corollary extends [2, Thm. 1.1] to the case of a product of norm forms. It generalises the unconditional version of [22, Cor. 1.6], a number of statements from [15, Section 4] and the main result of [18] in the case of the ground field $\mathbb{Q}$.

Corollary 1.3 Let $k_{1}, \ldots, k_{n}$ be number fields and let $m_{1}, \ldots, m_{n}$ be positive integers with $\operatorname{gcd}\left(m_{1}, \ldots, m_{n}\right)=1$. Let $L_{i} \in \mathbb{Q}\left[t_{1}, \ldots, t_{s}\right]$ be polynomials of degree 1 , for $i=1, \ldots, r$. Let $X$ be a smooth and proper variety over $\mathbb{Q}$ that is birationally equivalent to the affine hypersurface

$$
\prod_{i=1}^{r} L_{i}\left(t_{1}, \ldots, t_{s}\right)=\prod_{j=1}^{n} N_{k_{j} / \mathbb{Q}}\left(\mathbf{x}_{j}\right)^{m_{j}} .
$$

Then $X(\mathbb{Q})$ is dense in $X\left(\mathrm{~A}_{\mathbb{Q}}\right)^{\mathrm{Br}}$. 
Note that repetitions among $L_{1}, \ldots, L_{r}$ are allowed here. Corollary 1.3 is a particular case of Proposition 3.5 which deals with several equations like (2) and extends [2, Thm. 1.3].

This note consists of two sections. In $\S 2$ we make preliminary remarks, some of which, like Corollary 2.3, are not needed in the proof of our main results but could possibly be of independent interest. In $\S 3$ we prove Theorem 1.1, Corollary 1.3 and their generalisations.

Our proof of Theorem [1.1 uses descent like [3], [10] or [18], and not the fibration method like [22] or [15]. It was inspired by the approach of Colliot-Thélène and Sansuc [5] to degeneration of torsors of tori, and by their computation of universal torsors on conic bundles [6, Section 2.6]. We apply open descent based on Harari's formal lemma as in [9], with an improvement found in [8].

The author is grateful to Jean-Louis Colliot-Thélène for useful discussions over the past many years. I would like to thank Daniel Loughran for his question that led me to Proposition 2.2, and Olivier Wittenberg for useful comments.

\section{Reduction of varieties defined over a dicretely valued field}

Let $R$ be a discrete valuation ring with the field of fractions $K$, the maximal ideal $\mathfrak{m}$ and the residue field $\kappa=R / \mathfrak{m}$. We assume that $\kappa$ is perfect. Let $j: \operatorname{Spec}(K) \rightarrow$ $\operatorname{Spec}(R)$ and $i: \operatorname{Spec}(\kappa) \rightarrow \operatorname{Spec}(R)$ be the embeddings of the generic and the special points, respectively. We have an exact sequence of étale sheaves on $\operatorname{Spec}(R)$ :

$$
0 \rightarrow \mathbb{G}_{m, R} \rightarrow j_{*} \mathbb{G}_{m, K} \rightarrow i_{*} \mathbb{Z}_{\kappa} \rightarrow 0
$$

see [17, Examples II.3.9]. Let $T$ be a torus over $R$. Applying $\operatorname{Hom}_{R}(\widehat{T}, \cdot)$ we obtain an exact sequence of abelian groups

$$
H^{1}(R, T) \rightarrow H^{1}(K, T) \rightarrow \operatorname{Ext}_{\kappa}^{1}(\widehat{T}, \mathbb{Z}),
$$

see [21, p. 70] for a detailed proof. We note that $\operatorname{Ext}_{\kappa}^{1}(\widehat{T}, \mathbb{Z})=H^{1}\left(\kappa, \widehat{T}^{\circ}\right)$, where $T^{\circ}$ is the $\kappa$-torus dual to $T$, that is, such that $\widehat{T}^{\circ}=\operatorname{Hom}(\widehat{T}, \mathbb{Z})$ as Galois modules.

For an $R$-scheme $X$ we denote by $X_{K}$ and $X_{\kappa}$ the generic and special fibres of $X$, respectively.

Lemma 2.1 Let $Y$ be a $K$-torsor of $T_{K}$. Suppose that there is an integral normal scheme $X$ and a surjective morphism $X \rightarrow \operatorname{Spec}(R)$ with integral fibres such that the generic fibre $X_{K}$ is birationally equivalent to $Y$.

(i) Let $\kappa^{\prime}$ be the algebraic closure of $\kappa$ in the function field of the special fibre $\kappa\left(X_{\kappa}\right)$. Then the image of $[Y]$ in $H^{1}\left(\kappa, \widehat{T}^{\circ}\right)$ is in the kernel of the restriction map $H^{1}\left(\kappa, \widehat{T}^{\circ}\right) \rightarrow H^{1}\left(\kappa^{\prime}, \widehat{T}^{\circ}\right)$. 
(ii) If $X_{\kappa}$ is geometrically integral, then there is an $R$-torsor $Z$ of $T$ such that $Y \cong Z_{K}$

Proof. Let $R^{\prime}$ be the local ring of the special fibre $X_{\kappa}$. Since $X$ is normal and $X_{\kappa}$ is integral, $R^{\prime}$ is a discrete valuation ring. Since $X$ is integral, the field of fractions of $R^{\prime}$ is $K\left(X_{K}\right)$. A local parameter of $R$ is also a local parameter of $R^{\prime}$, because $X_{\kappa}$ is integral. Thus $\mathfrak{m}^{\prime}=\mathfrak{m} \otimes_{R} R^{\prime}$ is the maximal ideal of $R^{\prime}$, and the residue field $R^{\prime} / \mathfrak{m}^{\prime}$ is the field of functions on the special fibre $X_{\kappa}$.

The pullback from $R$ to $R^{\prime}$ gives rise to the commutative diagram

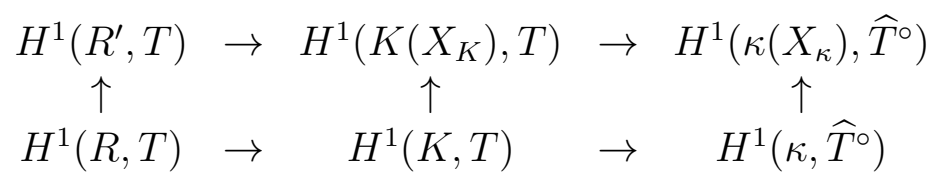

The restriction of the diagonal $Y \rightarrow Y \times_{K} Y$ to the generic fibre of $Y$ is a $K(Y)$ point of $Y$. Hence the torsor $Y$ is split by the field extension $K\left(X_{K}\right)=K(Y)$, so that the class $[Y] \in H^{1}(K, T)$ goes to zero in $H^{1}\left(K\left(X_{K}\right), T\right)$.

The right vertical map in the diagram factorises as follows:

$$
H^{1}\left(\kappa, \widehat{T}^{\circ}\right) \longrightarrow H^{1}\left(\kappa^{\prime}, \widehat{T}^{\circ}\right) \stackrel{\sim}{\longrightarrow} H^{1}\left(\kappa\left(X_{\kappa}\right), \widehat{T}^{\circ}\right) .
$$

The second map here is an isomorphism because $\widehat{T}^{\circ}$ is a finitely generated free abelian group and $\kappa^{\prime}$ is algebraically closed in $\kappa\left(X_{\kappa}\right)$. This implies (i).

If the $\kappa$-scheme $X_{\kappa}$ is geometrically integral, the field $\kappa$ is algebraically closed in $\kappa\left(X_{\kappa}\right)$, that is, $\kappa^{\prime}=\kappa$. Then $[Y]$ is in the image of the map $H^{1}(R, T) \rightarrow H^{1}(K, T)$, and this proves (ii).

The statement of Lemma 2.1 (i) leaves open the question to what extent $\kappa^{\prime}$ is determined by the field $K\left(X_{K}\right)$. We treat this as a question about integral, regular, proper schemes over a discretely valued field, see Corollary 2.3 below.

For an integral variety $V$ over a field $k$, we write $k_{V}$ for the algebraic closure of $k$ in $k(V)$.

Proposition 2.2 Let $Y$ and $Y^{\prime}$ be integral regular schemes that are proper over $R$. If there is a dominant rational map from $Y_{K}$ to $Y_{K}^{\prime}$, then for any irreducible component $C \subset Y_{\kappa}$ of multiplicity 1 there exists an irreducible component $C^{\prime} \subset Y_{\kappa}^{\prime}$ of multiplicity 1 such that $\kappa_{C^{\prime}} \subset \kappa_{C}$.

Proof. Write $F=K\left(Y_{K}\right)$ and $F^{\prime}=K\left(Y_{K}^{\prime}\right)$. We are given an inclusion $F^{\prime} \subset F$, or, equivalently, a morphism $\operatorname{Spec}(F) \rightarrow \operatorname{Spec}\left(F^{\prime}\right)$. Let $\mathcal{O}_{C}$ be the local ring of the generic point of $C$. Since $Y$ is regular, $\mathcal{O}_{C}$ is a discrete valuation ring. The multiplicity of $C$ is 1 , that is, the maximal ideal of $\mathcal{O}_{C}$ is $\mathfrak{m} \mathcal{O}_{C}=\mathfrak{m} \otimes_{R} \mathcal{O}_{C}$. Since $Y$ is integral, the field of fractions of $\mathcal{O}_{C}$ is $F$. The residue field of $\mathcal{O}_{C}$ is $\kappa(C)$. 
After the base change from $R$ to $\mathcal{O}_{C}$ we obtain a morphism $Y^{\prime} \times{ }_{R} \mathcal{O}_{C} \rightarrow \mathcal{O}_{C}$. Its generic fibre $Y_{F}^{\prime}=Y_{K}^{\prime} \times_{K} F$ has an $F$-point $\operatorname{Spec}(F) \rightarrow \operatorname{Spec}\left(F^{\prime}\right) \rightarrow Y_{F}^{\prime}$ coming from the $F^{\prime}$-point defined by the diagonal morphism $Y_{K}^{\prime} \rightarrow Y_{K}^{\prime} \times_{K} Y_{K}^{\prime}$. The morphism $Y^{\prime} \times{ }_{R} \mathcal{O}_{C} \rightarrow \mathcal{O}_{C}$ is proper, and by the valuative criterion of properness any $F$-point of its generic fibre extends to a section of the morphism.

Since $C$ has multiplicity 1, the special fibre of $Y^{\prime} \times{ }_{R} \mathcal{O}_{C} \rightarrow \mathcal{O}_{C}$ is $Y_{\kappa}^{\prime} \times{ }_{\kappa} \kappa(C)$. A section of $Y^{\prime} \times{ }_{R} \mathcal{O}_{C} \rightarrow \mathcal{O}_{C}$ thus gives rise to a $\kappa(C)$-point of $Y_{\kappa}^{\prime} \times_{\kappa} \kappa(C)$, which can be viewed also as a $\kappa(C)$-point of $Y_{\kappa}^{\prime}$. Since $Y^{\prime} \times{ }_{R} \mathcal{O}_{C}$ is regular, any section of $Y^{\prime} \times{ }_{R} \mathcal{O}_{C} \rightarrow \mathcal{O}_{C}$ meets the special fibre at a smooth point. In particular, this point belongs to exactly one geometric irreducible component, moreover, this component has multiplicity 1 (see the calculation in the proof of [20, Lemma 1.1 (b)], or [25, Lemme 3.8]).

Let $U \subset Y_{\kappa}^{\prime}$ be the smooth locus of $Y_{\kappa}^{\prime}$. Since $U$ contains a $\kappa(C)$-point, it is nonempty. By Stein factorisation, the structure morphism $U \rightarrow \operatorname{Spec}(\kappa)$ factors through the surjective morphism $U \rightarrow \operatorname{Spec}(L)$ with geometrically connected fibres, where $L$ is an étale $\kappa$-algebra. Explicitly, $L$ is the direct sum of finite field extensions of $\kappa$, each of which is the algebraic closure of $\kappa$ in the function field of an irreducible component of $Y_{\kappa}^{\prime}$ of multiplicity 1 . The image of the composed map $\operatorname{Spec}(\kappa(C)) \rightarrow \operatorname{Spec}(L)$ is connected, hence this is $\operatorname{Spec}\left(\kappa_{C^{\prime}}\right)$ for some irreducible component $C^{\prime} \subset Y_{\kappa}^{\prime}$ of multiplicity 1. Thus $\kappa_{C^{\prime}} \subset \kappa(C)$ and hence $\kappa_{C^{\prime}} \subset \kappa_{C}$.

Corollary 2.3 Let $X$ be an integral regular scheme that is proper over $R$. Let $\Sigma_{X}$ be the partially ordered set of irreducible components of multiplicity 1 of $X_{\kappa}$, where $C$ dominates $D$ if $\kappa_{D} \subset \kappa_{C}$. The set of finite field extensions $\kappa \subset \kappa_{C}$, where $C$ is a minimal element of $\Sigma_{X}$, is a birational invariant of the generic fibre $X_{K}$.

Proof. Suppose that proper $R$-schemes $X$ and $Y$ are integral and regular with birationally equivalent generic fibres, that is, $K\left(X_{K}\right) \cong K\left(Y_{K}\right)$. Let $C$ be a minimal element of $\Sigma_{X}$. By Proposition 2.2 there exists $C^{\prime} \in \Sigma_{Y}$ such that $\kappa_{C^{\prime}} \subset \kappa_{C}$. By the same proposition, there is $C^{\prime \prime} \in \Sigma_{X}$ such that $\kappa_{C^{\prime \prime}} \subset \kappa_{C^{\prime}}$. By minimality of $C$ we have $\kappa_{C^{\prime \prime}}=\kappa_{C}$, hence $\kappa_{C}=\kappa_{C^{\prime}}$. If $C^{\prime}$ is not minimal in $\Sigma_{Y}$, then, by Proposition 2.2. $C$ is not minimal in $\Sigma_{X}$.

This set of finite extensions of the residue field can be explicitly determined when the generic fibre is a conic, and, more generally, a Severi-Brauer variety, or a quadric of dimension 2 .

Remark. Olivier Wittenberg suggested a somewhat different approach to Proposition 2.2 and Corollary 2.3 . Consider a discrete valuation $v: K\left(X_{K}\right)^{*} \rightarrow \mathbb{Z}$ such that the restriction of $v$ to $K^{*}$ is the given discrete valuation of $K$. Let $R^{\prime}$ be the valuation ring of $v$, and let $\kappa^{\prime}$ be the algebraic closure of $\kappa$ in the residue field of $R^{\prime}$. Let us call $\Theta_{X}$ the resulting set of finite field extensions of $\kappa$ partially ordered by inclusion. It is clear that $\Theta_{X}$ is a birational invariant of $X_{K}$. The discrete valuation 
associated to an irreducible component of $X_{\kappa}$ of multiplicity 1 is an example of such a valuation, so we have an inclusion of partially ordered sets $\Sigma_{X} \subset \Theta_{X}$. Since $X$ is proper over $R$ and regular, it can be shown that any extension of the given discrete valuation of $K$ to $K\left(X_{K}\right)$ gives rise to a morphism of $R$-schemes $\operatorname{Spec}\left(R^{\prime}\right) \rightarrow X$ that factors through the smooth locus of $X / R$. Hence $\Sigma_{X}$ and $\Theta_{X}$ have the same set of minimal elements, which is thus a birational invariant of $X_{K}$.

\section{Torsors over toric fibrations}

Let $k$ be a field of characteristic zero. Let $\bar{k}$ be an algebraic closure of $k$, and let $\Gamma_{k}=\operatorname{Gal}(\bar{k} / k)$. For a variety $X$ over $k$ we write $\bar{X}=X \times_{k} \bar{k}$.

Let $T$ be a $k$-torus. We write $\widehat{T}$ for the $\Gamma_{k}$-module of characters of $T$.

Let $X$ be a smooth, proper, geometrically integral variety with a surjective morphism $f: X \rightarrow \mathbb{P}_{k}^{1}$ and the geometrically integral generic fibre $X_{k(t)}$ which is birationally equivalent to a $k(t)$-torsor of $T$.

Lemma 3.1 Each fibre of $X \rightarrow \mathbb{P}_{k}^{1}$ has an irreducible component of multiplicity 1.

Proof. The generic fibre of $\bar{X} \rightarrow \mathbb{P}_{\bar{k}}^{1}$ is birationally equivalent to a torsor of $\bar{T} \simeq \mathbb{G}_{m, \bar{k}}^{d}$, where $d=\operatorname{dim}(T)$. By Hilbert's theorem 90 we have $H^{1}(\bar{k}(t), T)=H^{1}\left(\bar{k}(t), \mathbb{G}_{m}\right)^{d}=$ 0 , so this torsor has a $\bar{k}(t)$-point. By the lemma of Lang and Nishimura, $X_{k(t)}$ has a $\bar{k}(t)$-point too. By the valuative criterion of properness, this point extends to a section of the proper morphism $\bar{X} \rightarrow \mathbb{P}_{\bar{k}}^{1}$. Since $X$ is smooth, by a standard argument (see the proof of [20, Lemma 1.1 (b)], or [25]) any section intersects each fibre of $\bar{X} \rightarrow \mathbb{P}_{\bar{k}}^{1}$ in an irreducible component of multiplicity 1 . The lemma follows.

Proof of Theorem 1.1. We keep the notation of $\S 1$. By Lemma 3.1 there is an irreducible component of multiplicity 1 in each $X_{P_{i}}$, for $i=1, \ldots, r$. We mark these components. We also mark a geometrically integral irreducible component of multiplicity 1 in each of the remaining fibres of $f$. Define $Y \subset X$ as the complement to the union of all the unmarked irreducible components of the fibres of $f: X \rightarrow \mathbb{P}_{k}^{1}$. It is clear that $Y$ is a dense open subset of $X$. The restriction of $f$ to $Y$ is a surjective morphism $f: Y \rightarrow \mathbb{P}_{k}^{1}$ with integral fibres, and with proper and geometrically integral generic fibre $Y_{k(t)}=X_{k(t)}$. It follows that $\bar{k}[Y]^{*}=\bar{k}^{*}$ and $\operatorname{Pic}(\bar{Y})$ is torsionfree.

Let $\operatorname{Pic}(\bar{Y}) \rightarrow \operatorname{Pic}\left(Y_{\bar{k}(t)}\right)$ be the homomorphism of $\Gamma_{k}$-modules induced by the inclusion of the generic fibre $Y_{\bar{k}(t)}$ into $\bar{Y}$. This homomorphism is surjective since $Y$ is smooth. Let $S$ be a $k$-torus defined by the exact sequence of $\Gamma_{k}$-modules

$$
0 \rightarrow \widehat{S} \rightarrow \operatorname{Pic}(\bar{Y}) \rightarrow \operatorname{Pic}\left(Y_{\bar{k}(t)}\right) \rightarrow 0
$$


Thus the abelian group $\widehat{S}$ is generated by the geometric irreducible components of the fibres $Y_{P_{1}}, \ldots, Y_{P_{r}}$. Recall that a vertical torsor $\mathcal{T} \rightarrow Y$ is a torsor of $S$ whose type is the injective map $\widehat{S} \rightarrow \operatorname{Pic}(\bar{Y})$ from (15). According to [21, Prop. 4.4.1] such torsors exist. For any vertical torsor we have $\bar{k}[\mathcal{T}]^{*}=\bar{k}^{*}$ and the abelian group

$$
\operatorname{Pic}(\overline{\mathcal{T}}) \cong \operatorname{Pic}\left(Y_{\bar{k}(t)}\right) \cong \operatorname{Pic}\left(X_{\bar{k}(t)}\right)
$$

is torsion-free. It follows that $\operatorname{Br}_{1}(\mathcal{T}) / \operatorname{Br}_{0}(\mathcal{T})$ is finite.

Recall that for $i=1, \ldots, r$ we write $k\left(P_{i}\right)$ for the residue field of $P_{i}$ and $k_{i}$ for the algebraic closure of $k\left(P_{i}\right)$ in the function field $k\left(Y_{P_{i}}\right)$. The variety $W_{\alpha}$ is defined by (1), which is also equation (4.34) of [21].

Lemma 3.2 The variety $W_{\alpha}$ is smooth and geometrically irreducible, and the morphism $\pi: W_{\alpha} \rightarrow \mathbb{P}_{k}^{1}$ given by $(u: v)$ is faithfully flat. We have $\bar{k}\left[W_{\alpha}\right]^{*}=\bar{k}^{*}$ and $\operatorname{Pic}\left(\bar{W}_{\alpha}\right)=0$.

Proof. The first statement is [21, Lemma 4.4.5]. The second statement is a straightforward adaptation of [6, Lemme 2.6.1].

Proposition 3.3 Any vertical torsor over $Y$ is birationally equivalent to $W_{\alpha} \times_{k} Z$, where $\alpha \in \prod_{i=1}^{r} k\left(P_{i}\right)^{*}$ and $Z$ is a $k$-torsor of $T$.

Proof. The local description of torsors due to Colliot-Thélène and Sansuc, see [21, Thm. 4.3.1, Cor. 4.4.6], can be stated as follows. Let

$$
U^{\prime}=\mathbb{A}_{k}^{1} \backslash\left\{P_{1}, \ldots, P_{r}\right\}, \quad U=Y \cap f^{-1}\left(U^{\prime}\right), \quad V_{\alpha}=\pi^{-1}\left(U^{\prime}\right) .
$$

Then for any vertical torsor $\mathcal{T} / Y$ there exists an $\alpha \in \prod_{i=1}^{r} k\left(P_{i}\right)^{*}$ such that the restriction $\mathcal{T}_{U}=\mathcal{T} \times_{Y} U$ is isomorphic to the fibred product $U \times_{U^{\prime}} V_{\alpha}$. Let us write $W=W_{\alpha}, V=V_{\alpha}$.

Let $j^{\prime}: \operatorname{Spec}(k(t)) \rightarrow U^{\prime}$ be the embedding of the generic point of $\mathbb{A}_{k}^{1}$ into the open set $U^{\prime}$. Let $i_{P}: P \rightarrow U^{\prime}$ be the embedding of a closed point $P \subset U^{\prime}$. Since $\mathbb{A}_{k}^{1}$ is smooth, there is the following exact sequence of étale sheaves on $U^{\prime}$ :

$$
0 \rightarrow \mathbb{G}_{m, U^{\prime}} \rightarrow j_{*}^{\prime} \mathbb{G}_{m, k(t)} \rightarrow \oplus_{P \in U^{\prime}} i_{P *} \mathbb{Z}_{k(P)} \rightarrow 0
$$

see [17, Examples II.3.9, III.2.22]. Similarly to the proof of Lemma 2.1, an application of $\operatorname{Hom}_{U^{\prime}}(\widehat{T}, \cdot)$ to this sequence produces an exact sequence

$$
H^{1}\left(U^{\prime}, T\right) \rightarrow H^{1}(k(t), T) \rightarrow \oplus_{P \in U^{\prime}} H^{1}\left(k(P), \widehat{T}^{\circ}\right) .
$$

Let $\xi \in H^{1}(k(t), T)$ be the class of a $k(t)$-torsor of $T$ birationally equivalent to $X_{k(t)}$. The map $H^{1}(k(t), T) \rightarrow H^{1}\left(k(P), \widehat{T}^{\circ}\right)$ in (6) can be computed in the local $\operatorname{ring} R=O_{P}$. The fibres of $f: U \rightarrow U^{\prime}$ are geometrically integral, hence Lemma 
2.1 (ii) implies that this map is trivial. Thus we see from (6) that $\xi$ comes from some $\xi^{\prime} \in H^{1}\left(U^{\prime}, T\right)$. By Lemma 2.1 (i) applied to $f: Y \rightarrow \mathbb{P}_{k}^{1}$ the image of $\xi^{\prime}$ in $H^{1}\left(k\left(P_{i}\right), \widehat{T}^{\circ}\right)$ goes to zero in $H^{1}\left(k_{i}, \widehat{T}^{\circ}\right)$.

The fibre $W_{i}=\pi^{-1}\left(P_{i}\right)$ is the product of a principal homogeneous space of a $k\left(P_{i}\right)$-torus and the affine $k\left(P_{i}\right)$-variety defined by $N_{k_{i} / k\left(P_{i}\right)}\left(z_{i}\right)=0$. Thus $W_{i}$ is integral over $k\left(P_{i}\right)$ and the field $k_{i}$ is the algebraic closure of $k\left(P_{i}\right)$ in the function field of $W_{i}$. If we set $P_{0}=\infty$, then $W_{0}=\pi^{-1}\left(P_{0}\right)$ is geometrically integral by construction, so the algebraic closure of $k\left(P_{0}\right)=k$ in $k\left(W_{0}\right)$ is $k_{0}=k$. We have a commutative diagram similar to the one in the proof of Lemma 2.1.

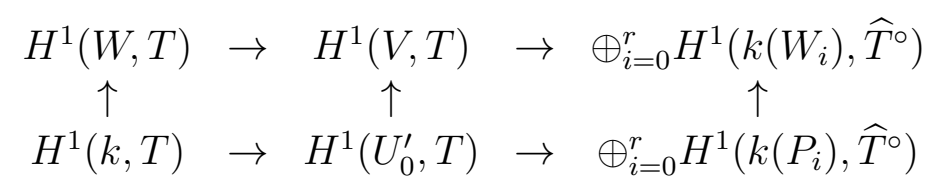

By the structure of degenerate fibres of $\pi: W \rightarrow \mathbb{P}_{k}^{1}$ described above, the image of $\xi^{\prime}$ in $H^{1}\left(k\left(W_{i}\right), \widehat{T}^{\circ}\right)$ is zero for $i=0, \ldots, r$. Now the upper sequence in the diagram shows that $\pi^{*}\left(\xi^{\prime}\right) \in H^{1}(V, T)$ comes from $H^{1}(W, T)$.

By Lemma 3.2 we have $\bar{k}\left[W_{\alpha}\right]^{*}=\bar{k}^{*}$ and $\operatorname{Pic}\left(\bar{W}_{\alpha}\right)=0$, and thus the fundamental exact sequence of Colliot-Thélène and Sansuc (see [6, Thm. 1.5.1] or [21, Cor. 2.3.9]) shows that the natural map $H^{1}(k, T) \rightarrow H^{1}(W, T)$ is an isomorphism. It follows that any $W$-torsor of $T$ is the product of $W$ and a $k$-torsor of $T$.

End of proof of Theorem 1.1. Let $\left(M_{v}\right) \in X\left(\mathrm{~A}_{k}\right)^{\mathrm{Br}}$. By a theorem of Grothendieck, $\operatorname{Br}_{1}(X)$ is naturally a subgroup of $\operatorname{Br}_{1}(Y)$. We have $\bar{k}[Y]^{*}=\bar{k}^{*}$, and this implies that $\operatorname{Br}_{1}(Y) / \operatorname{Br}_{0}(Y)$ is a subgroup of $H^{1}(k$, $\operatorname{Pic}(\bar{Y}))$, which is finite because $\operatorname{Pic}(\bar{Y})$ is torsion-free. Thus we can use [9, Prop. 1.1] (a consequence of Harari's formal lemma) which says that the natural injective map of topological spaces $Y\left(\mathrm{~A}_{k}\right)^{\operatorname{Br}_{1}(Y)} \rightarrow X\left(\mathrm{~A}_{k}\right)^{\operatorname{Br}_{1}(X)}$ has a dense image. Thus we can assume without loss of generality that $\left(M_{v}\right) \in Y\left(\mathrm{~A}_{k}\right)^{\operatorname{Br}_{1}(Y)}$.

The main theorem of the descent theory of Colliot-Thélène and Sansuc states that every point in $Y\left(\mathrm{~A}_{k}\right)^{\operatorname{Br}_{1}(Y)}$ is in the image of the map $\mathcal{T}_{0}\left(\mathrm{~A}_{k}\right) \rightarrow Y\left(\mathrm{~A}_{k}\right)$, where $\mathcal{T}_{0} \rightarrow Y$ is a universal torsor (see [6, Section 3] or [21, Thm. 6.1.2(a)]). Thus we can find a point $\left(N_{v}\right) \in \mathcal{T}_{0}\left(\mathrm{~A}_{k}\right)$ such that the image of $N_{v}$ in $Y$ is $M_{v}$ for all $v$.

The structure group of $\mathcal{T}_{0} \rightarrow Y$ is the Néron-Severi torus $T_{0}$ defined by the property $\widehat{T}_{0}=\operatorname{Pic}(\bar{Y})$. The dual of the injective map in (5) is a surjective morphism of tori $T_{0} \rightarrow S$. Let $T_{1}$ be its kernel. Then $\mathcal{T}=\mathcal{T}_{0} / T_{1}$ is a $Y$-torsor with the structure group $S$ whose type is the natural map $\widehat{S} \rightarrow \operatorname{Pic}(\bar{Y})$, so $\mathcal{T}$ is a vertical torsor. Since $\mathcal{T}_{0}$ is a universal torsor, we have $\bar{k}\left[\mathcal{T}_{0}\right]^{*}=\bar{k}^{*}$ and $\operatorname{Pic}\left(\overline{\mathcal{T}}_{0}\right)=0$, hence $\operatorname{Br}_{1}\left(\mathcal{T}_{0}\right)=\operatorname{Br}_{0}\left(\mathcal{T}_{0}\right)$. Let $\left(P_{v}\right) \in \mathcal{T}\left(\mathrm{A}_{k}\right)$ be the image of $\left(N_{v}\right)$. By the functoriality of the Brauer-Manin pairing we see that $\left(P_{v}\right) \in \mathcal{T}\left(\mathrm{A}_{k}\right)^{\operatorname{Br}_{1}(\mathcal{T})}$.

By Proposition 3.3 the variety $\mathcal{T}$ is birationally equivalent to $E \times W_{\alpha}$ for some $\alpha \in \prod_{i=1}^{r} k\left(P_{i}\right)^{*}$. Let $E^{c}$ be a smooth compactification of $E$. Then we have a 
rational map $g$ from the smooth variety $\mathcal{T}$ to the proper variety $E^{c}$, and a rational map $h$ from $\mathcal{T}$ to $W_{\alpha}$. By the valuative criterion of properness there is an open subset $\Omega \subset \mathcal{T}$ with complement of codimension at least 2 in $\mathcal{T}$ such that $g$ is a morphism $\Omega \rightarrow E^{c}$. Let $\Omega^{\prime} \subset \Omega$ be a dense open subset such that $(g, h)$ defines an open embedding $\Omega^{\prime} \subset E^{c} \times W_{\alpha}$.

By Grothendieck's purity theorem the natural restriction map $\operatorname{Br}(\mathcal{T}) \rightarrow \operatorname{Br}(\Omega)$ is an isomorphism. Thus $g^{*} \operatorname{Br}_{1}\left(E^{c}\right) \subset \operatorname{Br}_{1}(\Omega)$, so that $g^{*} \operatorname{Br}_{1}\left(E^{c}\right) \subset \operatorname{Br}_{1}(\mathcal{T})$. (This argument is borrowed from [9].)

Since $\operatorname{Br}_{1}(\mathcal{T}) / \operatorname{Br}_{0}(\mathcal{T})$ is finite, by a small deformation we can assume $P_{v} \in \Omega\left(k_{v}\right)$ for each $v$. Thus $\left(g\left(P_{v}\right)\right)$ is a well defined element of $E^{c}\left(\mathrm{~A}_{k}\right)^{\mathrm{Br}_{1}\left(E^{c}\right)}$. By Sansuc's theorem, $E(k)$ is then non-empty and, moreover, is dense in $E^{c}\left(\mathrm{~A}_{k}\right)^{\mathrm{Br}_{1}\left(E^{c}\right)}$.

Let $\Sigma$ be a finite set of places of $k$ containing all the places where we need to approximate. By assumption we can find a $k$-point in $\Omega^{\prime}$ that is arbitrarily close to $\left(g\left(P_{v}\right), h\left(P_{v}\right)\right)$ for $v \in \Sigma$. We conclude that there is a $k$-point in $\mathcal{T}$ that is arbitrarily close to $P_{v}$ for $v \in \Sigma$. The image of this point in $Y$ approximates $\left(M_{v}\right)$. This finishes the proof of Theorem 1.1 .

Proposition 3.4 Let $X$ be a smooth, proper, geometrically integral variety over $\mathbb{Q}$, and let $f: X \rightarrow \mathbb{P}_{\mathbb{Q}}^{n}$ be a surjective morphism satisfying the following properties.

(a) There is a torus $T$ over $\mathbb{Q}$ such that the generic fibre $X_{\mathbb{Q}(\eta)}$ of $f$ is birationally equivalent to a $\mathbb{Q}(\eta)$-torsor of $T \times_{\mathbb{Q}} \mathbb{Q}(\eta)$.

(b) There exist hyperplanes $H_{1}, \ldots, H_{r} \subset \mathbb{P}_{\mathbb{Q}}^{n}$ such that $f$ has split fibres at all points of codimension 1 of $\mathbb{P}_{\mathbb{Q}}^{n}$ other than $H_{1}, \ldots, H_{r}$.

Then $X(\mathbb{Q})$ is dense in $X\left(\mathrm{~A}_{\mathbb{Q}}\right)^{\mathrm{Br}}$.

Proof. We deduce this from a fibration theorem due to D. Harari [14, Thm. 3.2.1]. Choose a point $M \in \mathbb{P}_{\mathbb{Q}}^{n}(\mathbb{Q})$ such that the fibre $X_{M}$ is smooth. The projective lines through $M$ are in a natural bijection with the points of $\mathbb{P}_{\mathbb{Q}}^{n-1}$. Thus an appropriate open subset $V \subset X$ is a quasi-projective variety equipped with a surjective morphism $p: V \rightarrow \mathbb{A}_{\mathbb{Q}}^{n-1}$ with split fibres. Any point of $X_{M}(\overline{\mathbb{Q}})$ defines a section of $p$ over $\overline{\mathbb{Q}}$. The generic fibre of $p$ is birationally equivalent to a family of torsors of $T$ over an open subset of the projective line, hence it is geometrically integral and geometrically rational. In particular, a smooth and proper model of the generic fibre has trivial geometric Brauer group and torsion-free geometric Picard group. The fibres of $p$ over $\mathbb{Q}$-points of a non-empty open subset of $\mathbb{A}_{\mathbb{Q}}^{n-1}$ satisfy the assumptions of Corollary [1.2, hence all the conditions of [14, Thm. 3.2.1] are satisfied. An application of this result proves the proposition.

Proposition 3.5 Let $k_{1}, \ldots, k_{n}$ be number fields, and let $L_{i} \in \mathbb{Q}\left[t_{1}, \ldots, t_{s}\right]$ be polynomials of degree 1 , for $i=1, \ldots, r$. Let $m_{i j} \geq 0$, for $i=1, \ldots, \ell$ and $j=1, \ldots, n$, be integers such that the sublattice of $\mathbb{Z}^{n}$ generated by the rows of the matrix $\left(m_{i j}\right)$ 
is primitive. Finally, let $d_{h i} \geq 0$ be integers, where $h=1, \ldots, \ell$ and $i=1, \ldots, r$. Let $X$ be a smooth and proper variety over $\mathbb{Q}$ that is birationally equivalent to the affine variety given by the system of equations

$$
c_{h} \prod_{i=1}^{r} L_{i}\left(t_{1}, \ldots, t_{s}\right)^{d_{h i}}=\prod_{j=1}^{n} N_{k_{j} / \mathbb{Q}}\left(\mathbf{x}_{j}\right)^{m_{h j}}, \quad h=1, \ldots, \ell
$$

where $c_{h} \in \mathbb{Q}^{*}$. Then $X(\mathbb{Q})$ is dense in $X\left(\mathrm{~A}_{\mathbb{Q}}\right)^{\mathrm{Br}}$.

Proof. The condition on the matrix $\left(m_{i j}\right)$ implies that the affine variety given by

$$
\prod_{j=1}^{n} N_{k_{j} / \mathbb{Q}}\left(\mathbf{x}_{j}\right)^{m_{h j}}=1, \quad h=1, \ldots, \ell
$$

is a torus. Let us call it $T$. The affine variety $Y$ given by (17) has a morphism $g: Y \rightarrow \mathbb{A}_{\mathbb{Q}}^{s}$ given by the coordinates $t_{1}, \ldots, t_{s}$. Let $H_{i}$ be the hyperplane $L_{i}=0$. The restriction of $g$ to $\mathbb{A}_{\mathbb{Q}}^{s} \backslash\left(H_{1} \cup \ldots \cup H_{r}\right)$ is a torsor of $T$. We can choose a smooth compactification $X$ of the smooth locus $Y_{\text {sm }}$ in such a way that there is a surjective morphism $f: X \rightarrow \mathbb{P}_{\mathbb{Q}}^{s}$ extending $g: Y_{\text {sm }} \rightarrow \mathbb{A}_{\mathbb{Q}}^{s}$. Now we apply Proposition 3.4.

For $\ell=1$ this statement is Corollary 1.3 .

\section{References}

[1] T. Browning and D.R. Heath-Brown. Quadratic polynomials represented by norm forms. GAFA 22 (2012) 1124-1190.

[2] T. Browning and L. Matthiesen. Norm forms for arbitrary number fields as products of linear polynomials. arxiv1307.7641

[3] T. Browning, L. Matthiesen and A.N. Skorobogatov. Rational points on pencils of conics and quadrics with many degenerate fibres. arXiv:1209.0207

[4] J.-L. Colliot-Thélène and P. Salberger. Arithmetic on some singular cubic hypersurfaces. Proc. London Math. Soc. 58 (1989) 519-549.

[5] J.-L. Colliot-Thélène and J.-J. Sansuc. Principal homogeneous spaces under flasque tori: applications. J. Algebra 106 (1987) 148-205.

[6] J.-L. Colliot-Thélène et J.-J. Sansuc. La descente sur les variétés rationnelles, II. Duke Math. J. 54 (1987) 375-492.

[7] J.-L. Colliot-Thélène, J.-J. Sansuc and Sir Peter Swinnerton-Dyer. Intersections of two quadrics and Châtelet surfaces, I, II. J. reine angew. Math. 373 (1987) $37-168$. 
[8] J.-L. Colliot-Thélène, D. Harari et A.N. Skorobogatov. Valeurs d'un polynôme à une variable représentées par une norme, Number theory and algebraic geometry, London Math. Soc. Lecture Note Ser. 303. Cambridge University Press, 2003, pp. 69-89.

[9] J.-L. Colliot-Thélène and A.N. Skorobogatov. Descent on fibrations over $\mathbb{P}_{k}^{1}$ revisited. Math. Proc. Camb. Phil. Soc. 128 (2000) 383-393.

[10] U. Derenthal, A. Smeets and D. Wei. Universal torsors and values of quadratic polynomials represented by norms. arXiv:1202.3567

[11] B. Green and T. Tao. Linear equations in primes. Ann. Math. 171 (2010) 17531850 .

[12] B. Green and T. Tao. The Möbius function is strongly orthogonal to nilsequences. Ann. Math. 175 (2012) 541-566.

[13] B. Green, T. Tao and T. Ziegler. An inverse theorem for the Gowers $U^{s+1}[N]$ norm. Ann. Math. 176 (2012) 1231-1372.

[14] D. Harari. Flèches de spécialisation en cohomologie étale et applications arithmétiques. Bull. Soc. Math. France 125 (1997) 143-166.

[15] Y. Harpaz, A.N. Skorobogatov and O. Wittenberg. The Hardy-Littlewood conjecture and rational points. arXiv:1304.3333

[16] R. Heath-Brown and A.N. Skorobogatov. Rational solutions of certain equations involving norms. Acta Math. 189 (2002) 161-177.

[17] J.S. Milne. Étale cohomology. Princeton University Press, 1980.

[18] D. Schindler and A.N. Skorobogatov. Norms as products of linear polynomials. J. London Math. Soc., to appear. arXiv:1210.5727

[19] A.N. Skorobogatov. Arithmetic on certain quadric bundles of relative dimension 2, I. J. reine angew. Math. 407 (1990) 57-74.

[20] A.N. Skorobogatov. Descent on fibrations over the projective line. Amer. J. Math. 118 (1996) 905-923.

[21] A. Skorobogatov. Torsors and rational points. Cambridge University Press, 2001.

[22] A. Smeets. Principes locaux-globaux pour certaines fibrations en torseurs sous un tore. Math. Proc. Camb. Phil. Soc., to appear. arXiv:1305.0756 
[23] M. Swarbrick Jones. A note on a theorem of Heath-Brown and Skorobogatov. Quat. J. Math. 64 (2013) 1239-1251.

[24] Sir Peter Swinnerton-Dyer. Rational points on some pencils of conics with 6 singular fibres. Ann. Fac. Sci. Toulouse 8 (1999) 331-341.

[25] O. Wittenberg. Intersections de deux quadriques et pinceaux de courbes de genre 1. Lecture Notes Math. 1901, Springer-Verlag, 2007.

Department of Mathematics, South Kensington Campus, Imperial College London, SW7 2BZ England, U.K. - and - Institute for the Information Transmission Problems, Russian Academy of Sciences, 19 Bolshoi Karetnyi, Moscow, 127994 Russia

a.skorobogatov@imperial.ac.uk 